\title{
Optimization of digitization procedures in cultural heritage preservation
}

\author{
Bea Martínez ${ }^{\mathrm{a}}$, Carles Mitjà ${ }^{\mathrm{a}}$, Jaume Escofet ${ }^{\mathrm{b}}$ \\ ${ }^{a}$ Image Quality Laboratory/CITM/UPC, Campus de Terrassa, Edif. TR12, 08222-Terrassa, \\ Barcelona, Spain \\ ${ }^{\mathrm{b}}$ Dept. of Optics and Optometry/UPC, Campus de Terrassa, Edif. TR8, 08222-Terrassa, Barcelona, \\ Spain
}

\begin{abstract}
The digitization of both volumetric and flat objects is the nowadays-preferred method in order to preserve cultural heritage items. High quality digital files obtained from photographic plates, films and prints, paintings, drawings, gravures, fabrics and sculptures, allows not only for a wider diffusion and on line transmission, but also for the preservation of the original items from future handling. Early digitization procedures used scanners for flat opaque or translucent objects and camera only for volumetric or flat highly texturized materials. The technical obsolescence of the high-end scanners and the improvement achieved by professional cameras has result in a wide use of cameras with digital back to digitize any kind of cultural heritage item. Since the lens, the digital back, the software controlling the camera and the digital image processing provide a wide range of possibilities, there is necessary to standardize the methods used in the reproduction work leading to preserve as high as possible the original item properties. This work presents an overview about methods used for camera system characterization, as well as the best procedures in order to identify and counteract the effect of the lens residual aberrations, sensor aliasing, image illumination, color management and image optimization by means of parametric image processing. As a corollary, the work shows some examples of reproduction workflow applied to the digitization of valuable art pieces and glass plate photographic black and white negatives.
\end{abstract}

Keywords: cultural heritage, digitization, characterization, Dynamic Range, MTF, aliasing, color accuracy.

\section{INTRODUCTION}

Digitization of cultural heritage items is becoming a prominent activity in museums, photographic archives, and institutions preserving paintings, drawings and/or photographs. Those institutions have the challenge to preserve valuable items alongside its maximum public availability. Nowadays, the Information and Communications Technologies (ICT) are of great importance as a mean of diffusion and accessibility. The digitization has the advantage allowing for diffusion as well as avoiding unnecessary further handling. A digitization plan must warrant that digital images reproduce the original items with the maximum accuracy and must accomplish with some characteristics that enable them to be optimized for different transmission and visualizing systems. Moreover, the huge quantity of items that museums and archives are managing implies the need of digital repositories with high capacity. So, the balance between good quality and a low files volume must be achieved. There are some crucial steps to be performed before starting a digitization process, including the choice of the optimum settings in relation to the item's properties by means of the capture system performance characterization, the experimental set up definition and the suitable image post-processing.

Early digitization procedures used scanners for flat opaque or translucent objects and camera only for volumetric or flat highly texturized materials. The technical obsolescence of the high-end scanners and the improvement achieved by professional cameras has entailed in a wide use of digital-back cameras to digitize any kind of cultural heritage item. Additionally, camera avoids any physical contact with delicate items, provides the use of a wide range of lighting sources that can contribute to a better original features reproduction and avoids any excess of heat spoiling some originals in fragile conditions. Cameras should be characterized in order to establish its performance reproducing originals with a wide variety of properties. 
This work proposes different optics and camera characterization procedures focusing on the digitization of opaque and transparent flat items. Several devices with different lens and sensor combinations, as well as some operation modes are assessed to establish the optimum performance for each combination thereof. In order to achieve this aim, a set of suitable test targets related with lens aberrations, dynamic range and noise, modulation transfer function (MTF) and color management have been captured with the same working conditions, especially those affecting image magnification and lighting schemes. Results obtained after processing information derived from this images help to establish the system settings that perform the optimum reproduction procedures.

\section{MATERIALS AND METHODS}

\subsection{Lighting for digitization}

Lighting requirements in reproduction tasks are quite different depending on the properties of the original. Drawings and photographic prints are flat opaque while negative and reversal materials are flat translucent. The procedure with flat opaque items is different according to its physical size. Items up to DIN-A3 size can be reproduced in a vertical copy stand. This device allows for original flatness even pressing with glass when necessary and facilitates the proper parallelism between the original, lens and sensor planes. The bigger sized originals must be reproduced with the optical axis in horizontal direction. This setup complicates the accurate alignment of the camera position related to the original surface and special devices must be employed to avoid a shape distortion of the original caused by unparalleled set up [1].

In both cases, the originals must be evenly illuminated avoiding light sources reflections over its surface, specially those with specular or glossy properties. The most useful schemes use two lamps, one on each side, or four lamps at four original corners. The evenness of illumination must be accurately set up with photometric measurements over the original plane and/or checked by image processing techniques from a test target image [1]. Working with opaque textured materials, lighting can be used to enhance the texture properties, placing a unique light fixture in an appropriate direction. Digital image processing can solve unevenness provoked by such a lighting scheme.

In translucent materials as photographic negatives or positive slides, transmitted light must be used. Because of the granular structure of those materials, some prevention must be taken in lighting system properties due to light scattering. This granular structure provokes different light scattering depending on optical density; moreover, that is a property of each type of photographic emulsion and is characterized by the so-called Callier factor or Q factor. Being proven that the $\mathrm{Q}$ factor is almost constant beyond a density of 0.3 , the effective transmitted density and hence the image contrast depends only on the illumination specularity [1]. Furthermore, diffused light and the consequent amount of scattering reduces original contrast and tends to subdue some little marks and scratches present on damaged originals. Then, the most useful transmission system to illuminate this kind of originals is diffuse transmission. Those problems do not affect the dyed materials as color films or monochrome chromogenically developed films that can be indifferently illuminated with specular or diffused transmitted light [2].

A suitable illuminant for reproduction work must accomplish a set of requirements as: a) Power enough for sensor sensitivity at as short as possible exposure time, b) the closest color temperature to one of the several standard camera available settings, c) equalized spectral distribution over the visible range, d) constant power output over time and e) minimum heat so as not to cause damage on valuable items. Although some of those properties can be accomplished by several available photographic lighting systems, the best overall performance is provided by electronic flash strobes. The electronic flash is extremely constant along the useful tube life regarding both power and spectral properties and its color temperature is specifically intended in all high-end camera settings. The short duration of the power output, always below $1 / 200$ s diminishes unexpected camera shaking effects and harmful radiation accumulation over delicate items.

\subsection{Optical system testing}

Optical systems characterization is usually achieved by means of aberrometers, but this is complex out of specialized facilities. Therefore, in order to identify the presence of residual optical aberrations, the test target shown in Figure 1 is photographed through the whole diaphragm apertures range, while maintaining the same exposure and magnification as those used in the digitization process.

The structure of the test target (Figure 1.a) is based on the repetition of cross-shaped black figures over a white background, distributed in a radial direction from the center to the corners of the image. This distribution and the fact that the crosses are oriented in radial and transversal directions, allow detecting transversal chromatic aberration (TCA) and astigmatism. TCA generates color fringes along the tangential edges while astigmatism produces different blurring in 
tangential and radial directions. In both cases, these aberrations increase with radial distance to the center. Besides, this test target allows analyzing the presence of the combined effects of residual spherical aberration, field curvature and coma, as well as the presence of distortion, that can be identified by checking the straightness of the vertical and horizontal lines. The results of this characterization are useful to choose the must suitable lens to work with. The images of the target test are visually evaluated and digitally analyzed; the profile plots of the tangential and radial edges (Figure 1.b), at the center and the corners of the image, are obtained to compare their sharpness.

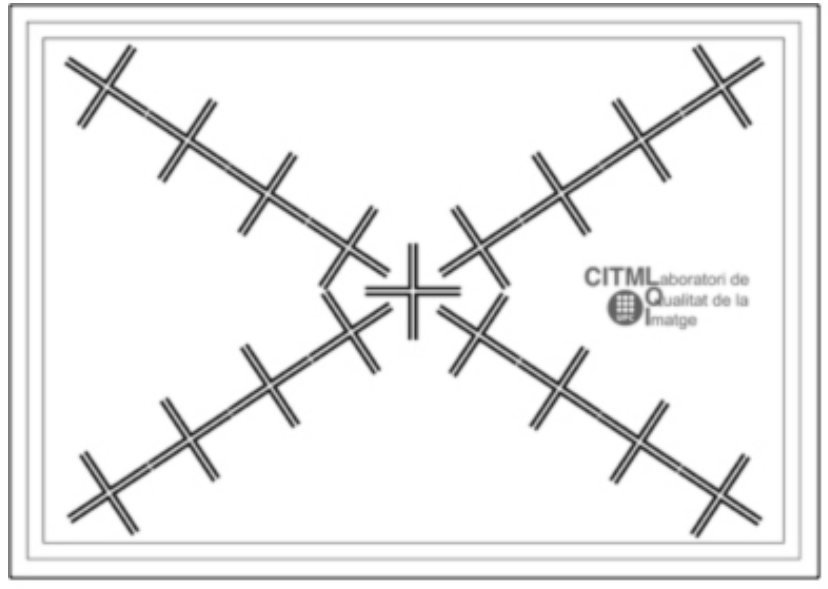

a)

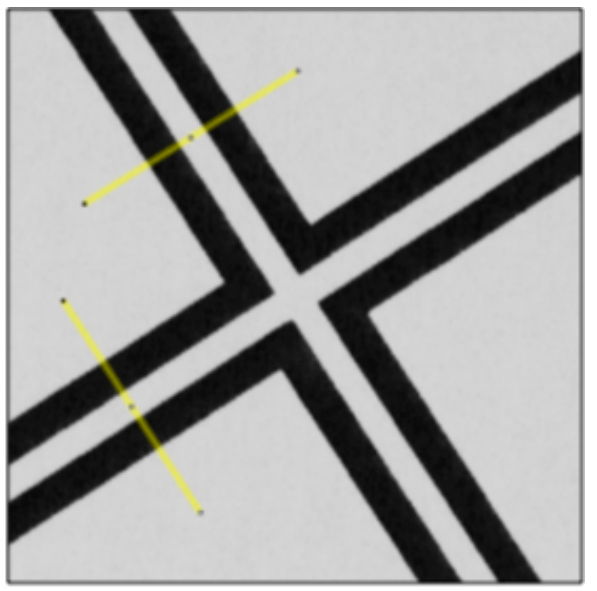

b)

Fig. 1. a) Test target that enables to evaluate the presence of residual optical aberrations. b) Magnified section of the test. The yellow lines indicate the pixel selections from which the tangential and radial profile plots will be obtained.

\subsection{Dynamic Range}

The original items to be reproduced are composed by a range of luminances that should be transferred to the image and this is why the capture system must be able to encompass the object dynamic range. In order to verify it, the system optoelectronic conversion function (OECF) reports about the useful dynamic range (DR). For practical purposes, the system DR is expressed in EV values and, in reproduction work, the most extended object DR can be found in photographic black and white negative materials, up to an optical density (OD) of 3.0 units and exceptionally, up to 4.0 OD units with high contrast color slides; 3.0 OD units equals to a DR of 10EV. Therefore, the test target used for the OECF measurement must exceed this dynamic range. This can be achieved by using the Stouffer T4110 step wedge (Figure 2), which holds a grey scale equivalent to $4.10 \mathrm{D}$ or $13.6 \mathrm{EV}$.

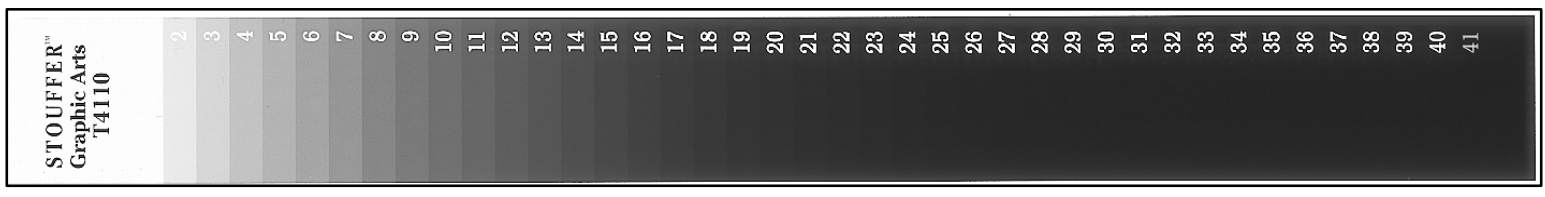

Fig. 2. Stouffer T4110 step wedge.

A plot of logarithm of gray values, taken from each test target image patch, against OD is the system OECF under the working conditions. The last useful patch criteria is appointed by a minimum Signal to Noise Ratio (SNR) of $1 \mathrm{~dB}$ [3]. The SNR of all the patches of the Stouffer T4110 pattern is calculated by measuring its gray value average and standard deviation. Moreover, the OECF plot allows checking the departure from linearity applied by the several process options and/or camera settings. 


\subsection{System MTF}

The system MTF is measured by the Slanted Edge method [4]. The image of a slanted edge test target (Figure 3) is captured for all the available diaphragm apertures, under the specific working conditions. The MTF measurement is performed by means of the ImageJ plugin SE_MTF_2xNyquist [5]. The structure of the test target offers the possibility of measuring the MTF both in horizontal and vertical directions from the same image file; this procedure avoids the variations that can occur when capturing sequential images, even under the same conditions. The text area is used to center the autofocus (AF) system measurement zone and the $25 \mathrm{~mm}$ ruler is useful to calculate the effective magnification of the image.

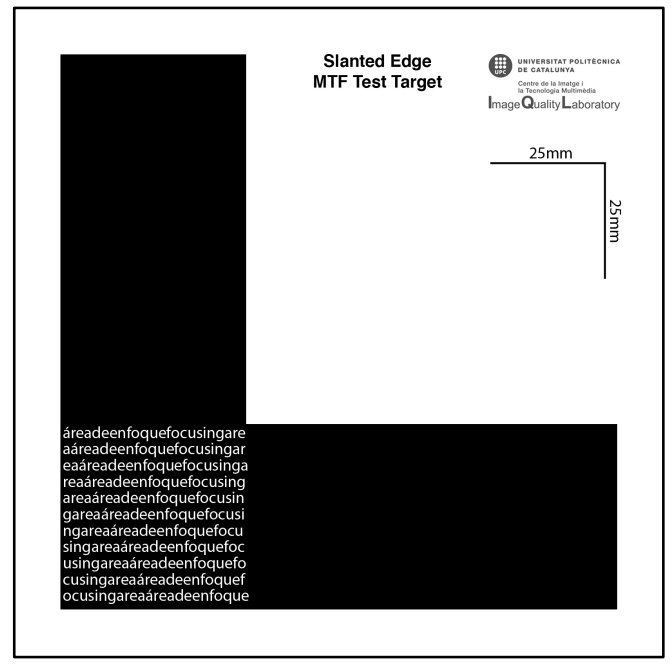

Fig. 3. Test target for the measurement of the system MTF.

MTF values, in addition to those of the optical system testing, allow establishing the most useful aperture range, regarding the effects of both aberrations and diffraction. In the case of cameras offering different capture or processing options that are worth to be evaluated, the series of images is obtained also for each of these operation modes. This is applied, for example, to systems that can perform demosaicing process with or without color interpolation.

As a corollary of the system MTF measurement with the ImageJ plugin SE_MTF_2xNyquist, the ESF plot can be used to assess the application of edges enhancement algorithms during the raw processing, avoiding the introduction of visual artifacts around the edges due to an excessive edge enhancement.

\subsection{Aliasing analysis}

For high diaphragm apertures, the lens transmits higher frequencies than the sensor Nyquist limit. Moreover, all high-end digital backs do not equip any kind of antialiasing low pass filter and consequently, aliasing artifacts may appear. This is observed, particularly, in digital sensors of high-end camera backs equipped with a Bayer RGB color filter array. In these devices the reproduction of edges or high frequency periodic patterns leads, in some cases, to the appearance of color aliasing artifacts, known as moiré patterns [6]. Even in those cases where the original content is randomly distributed, aliased frequencies can introduce messy artifacts in the image.

The system response to the aliasing phenomenon is carried out by the image analysis of a series of sinusoidal patterns of increasing frequency in the transmission sinusoidal target $(0.375$ to $801 \mathrm{p} / \mathrm{mm})$ supplied by Edmund Optics (Figure 4). For the digitization of the test, the working magnification must be adjusted in order to match a given test pattern with the sensor Nyquist frequency. The images of the test target are taken for all the available diaphragm apertures and, if needed, for the different sensor operation modes. 


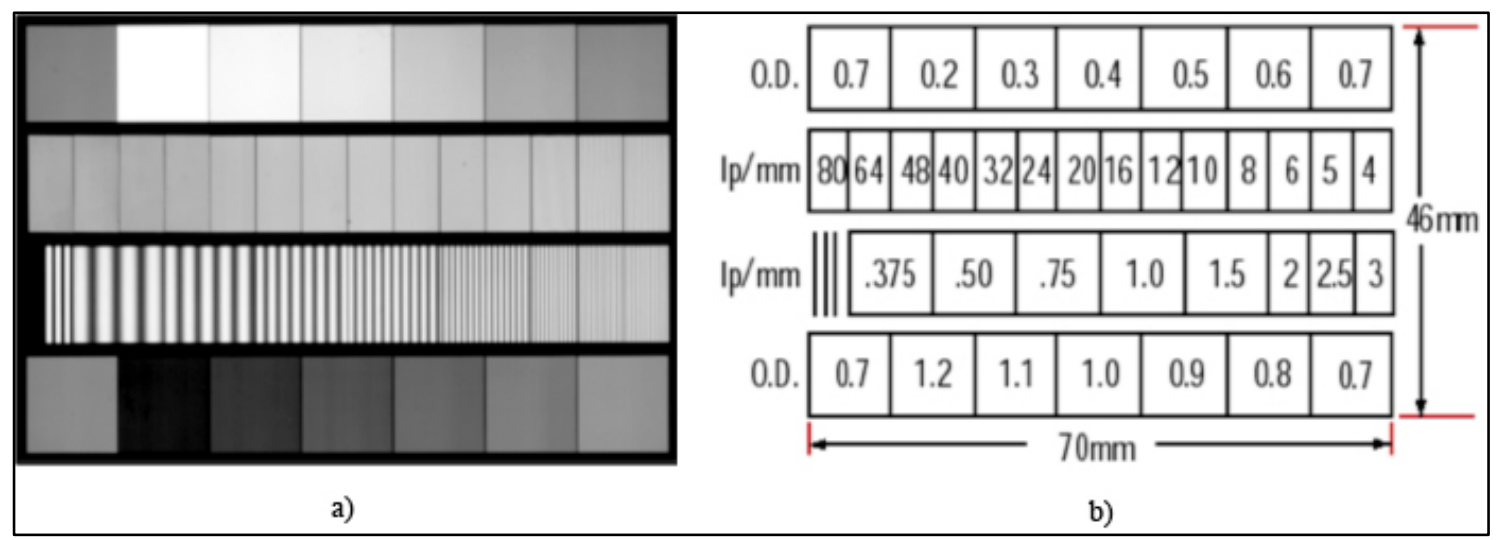

Fig. 4. a) Image of the sinusoidal test used for the measurement of the system response in terms of aliasing; b) Test scheme showing dimensions, the different pattern frequencies and the optical density of the step wedge incorporated.

The image analysis for measuring the aliasing effects begins by a visual evaluation, in order to detect the eventual occurrence of colored moiré patterns. At this point, it is relevant to take into account that the test target is completely monochromatic, so the presence of colored patterns is due to the specific raw processing. Intensity plot profiles in gray levels for the patches with Nyquist frequency, two beyond and two below, are analyzed. In the case of aliased images, patches with higher frequency than Nyquist are displayed with lower frequency.

\subsection{Color management}

Color reproduction is a critical feature on cultural heritage items digitization, even for monochrome originals, because it affects the three perceptual color components: hue, saturation and lightness. The application of specific International Color Consortium (ICC) profiles improves not only the color accuracy but also shadows reproduction and general contrast. Furthermore, it is important to take into account that the representation and optimization of color depend on ICC color management as well as on some processing settings like white balance and the tone curve.

Some of the most used raw processing software supplies the generic ICC profiles (or non standard format profiles as in Adobe Camera Raw) for each camera brand and model, but it is possible to obtain better results by creating the specific ICC profile for the given working conditions. As color reproduction depends on any fixture physical property as well as additional diffusers and or filters, glass panels used to flat the original item, sensor configuration, etc, the digitization of the IT8.7 test chart (Figure 5) for the creation of the ICC profile must be performed for the different capture settings applying to the different original properties. The obtained images of the IT8.7 contain the information that, together with the color values of the original test chart, ICC profiling software needs to generate the ICC profile of the system; the one used in this work is the Profile Maker.

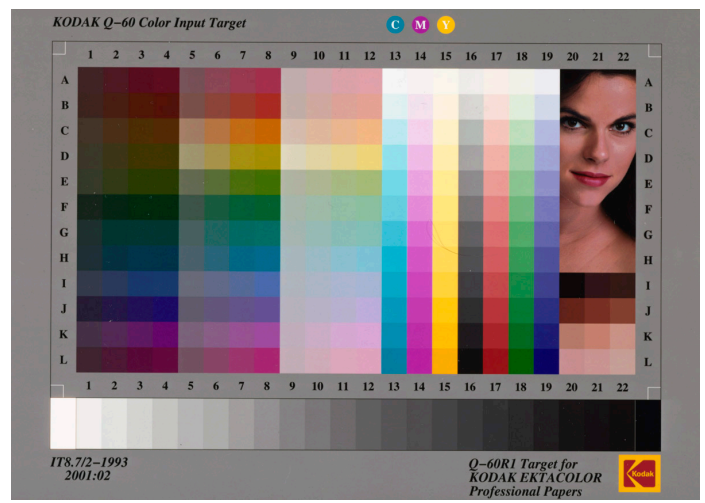

Fig. 5. IT8.7/2 color test chart for the creation of the camera ICC profiles. The test is available in two versions suitable for transparent materials (IT8.7/1) and opaque originals (IT8.7/2). 
Finally, the level of color accuracy achieved with the application of the ICC profiles in a color-managed workflow is of special relevance in this kind of works, especially for the possibility of using the resulting images for further analysis or data extraction [6]. The theoretical improvements of color management must be verified as a part of the characterization and optimization process. This verification is carried out by processing the image of the test chart under different conditions and color management options. The Lab values taken from the color patches of the obtained images are measured and compared with the Lab values of the original test chart by calculating the $\Delta \mathrm{E}$ value for each color patch. In order to obtain single value to compare the level of color accuracy, the average $\Delta \mathrm{E}$ for all the color patches of the test chart as well as the standard deviation are calculated.

\section{CASE STUDY EXAMPLES}

The results provided by the procedures described in section 2 are related with relevant aspects that should be analyzed and managed in order to obtain accurate and useful digital archives when digitizing cultural heritage items. The scope here is to show some example results, extracted from different case studies, which illustrate the kind of relevant information that can be obtained by these methods. This information may be useful to take decisions about equipment choice, capture settings and/or image processing options.

\subsection{Optical system testing}

Figure 6 shows a square selection of the corner of a test target image in which TCA effects can be observed. Red and cyan fringes are visible along the tangential edges, as confirmed by the RGB values measured at these zones. As TCA can be partially corrected by image processing, if no other lens without aberrations was available, this lens could be used by establishing a suitable correction algorithm.

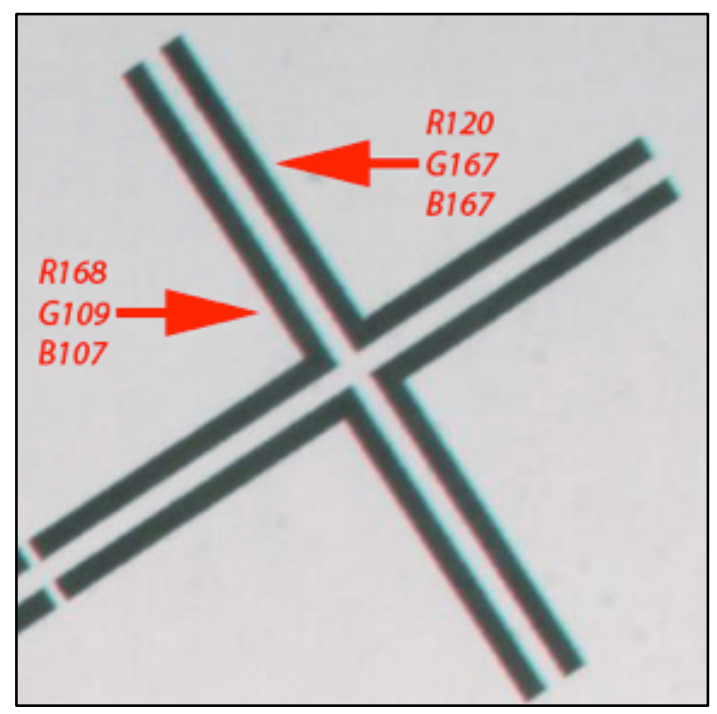

Fig. 6. Section of a corner of the residual aberrations detection test target image. In red, RGB composition of the coloured fringes caused by the presence of residual TCA.

\subsection{Dynamic range}

Figure 7 shows the OECF's related to two images of the Stouffer T4110 step wedge test. They have been obtained with a PhaseOne 645DF Camera equipped with a P40+ digital back and a PhaseOne120mm AF Macro f/4 lens, applying different processing settings. The image that displays the curve of figure 7.a has been processed with a non-linear tone curve (denoted as film curve by the raw processing software) and a generic ICC profile provided by the camera software, while the shown in the Figure 7.b was processed with no gamma application, the so called linear setting in the software controlling the raw processing, in addition to a specific ICC profile. 
In both cases, DR is around 3.1 OD units. This means that the system is able to encompass the maximum possible DR of the majority of photographic items to be digitized, which ranges up to $2.3 \mathrm{OD}$ for B\&W paper and 2.8 OD for most common B\&W negatives. Even with those materials that can overcame this density range, as some high contrast color slides, rising up to 4.0 OD units [2] the precise knowledge of the capture device DR allows for take bracketed exposure strategies to capture the whole item DR. However, the curves shape in the range between 0 and 2.0 OD units are significantly different in terms of linearity; it can be confirmed that the image processed with a linear tone curve performs also a more linear OECF, specially for the higher lightness values, which are the most critical for a correct tone reproduction. In this case, the use of a linear curve for raw files processing should be recommended.

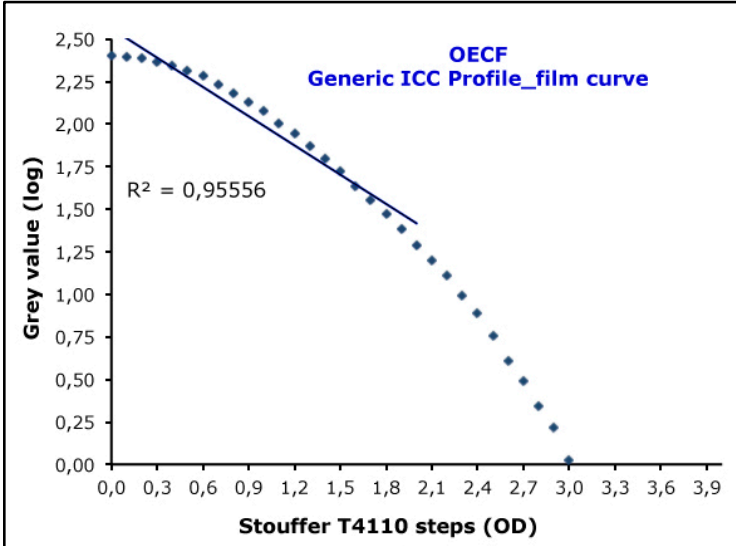

a)

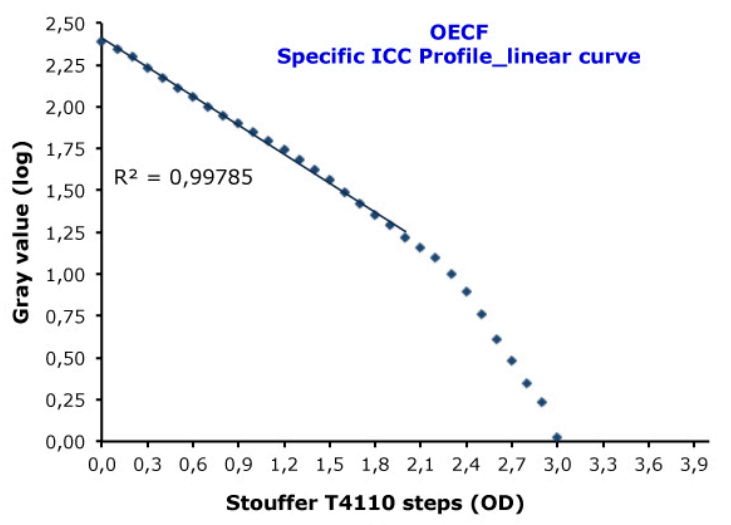

b)

Fig. 7. OECF curves from two images of Stouffer T4110 step wedge test processed with different tone curves and ICC profiles (dashed lines). Superimposed, respective linear regression curves (solid lines) for step densities up to 2.0 OD units. (a) Image processed with a non-linear curve and a generic ICC profile. (b) Image processed with a linear curve and a specific ICC profile.

\subsection{System MTF}

In this case study, the MTF of two different sensor configurations in the same system as in section 3.2 has been calculated: the first one applies a color interpolation algorithm in the demosaicing process (Standard mode) and the other one makes a half demosaicing, obtaining one image pixel from the RGB information of four photo detectors. One Red, two Green and one Blue photo detectors form each group following the Bayer scheme. This operation mode receives the name of Sensor+. The Figure 8 shows the MTF's of both configurations for all the available diaphragm apertures. It can be observed that the Standard mode achieves a higher resolution (because its Nyquist limit is twice the frequency of the Sensor + mode) and reproduces also a greater contrast. Moreover, the system performance in terms of sharpness is clearly dependent on the diaphragm aperture; for $\mathrm{f} / 4$ the effects of residual aberrations are noticeable, while diffraction reduces image quality significantly for $\mathrm{f} / 32$. With both operation modes, the optimum results are obtained for $\mathrm{f} / 8$ and $\mathrm{f} / 11$ diaphragm apertures. 


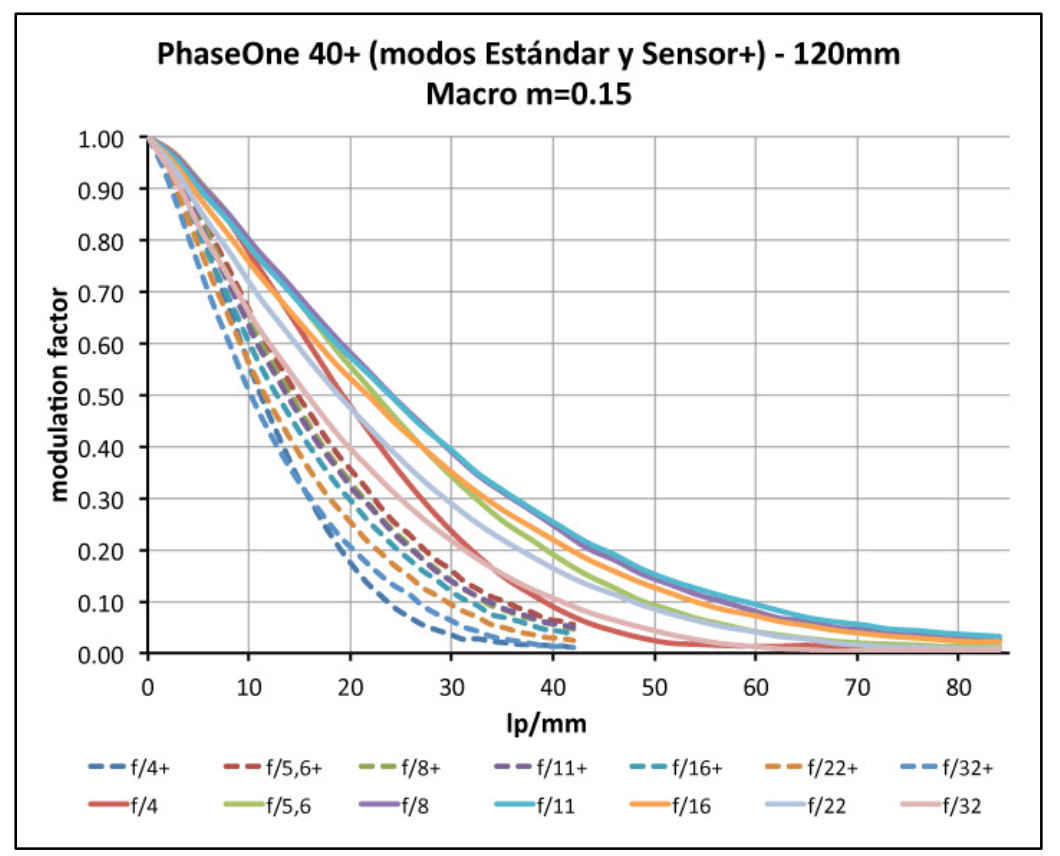

Fig. 8. Series of system MTF calculated for the Standard and Sensor+ modes (solid and dashed lines, respectively) and for all the available diaphragm apertures.

\subsection{Optimizing edges enhancement}

The ESF curves provided by the ImageJ plugin SE_MTF_2xNyquist can be used to control the edge enhancement processing. By analyzing the curve shape at both bounds of the ESF slope it is possible to detect the presence of artifacts along edges. Figure 9.a shows an ESF of a raw image without edge enhancement processing; the transition bounds are round shaped, which indicates a smooth edge. When applying the optimized edge enhancement the transition bounds show a sharper edge (Figure 9.b), while applying an excessive enhancement the artifacts appear as local maximum and minimum in the transition bounds (Figure 9.c). The most critical peak in terms of visual perception is that of the maximum grey values.

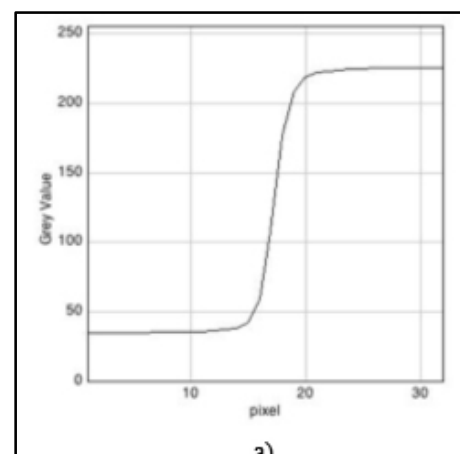

a)

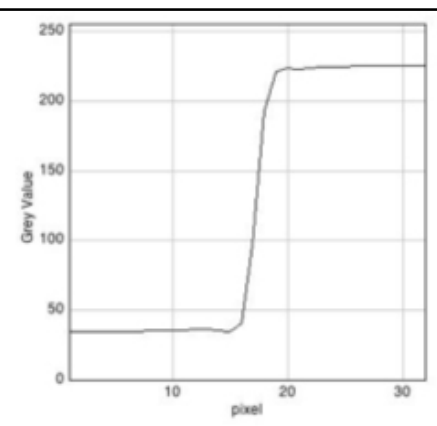

b)

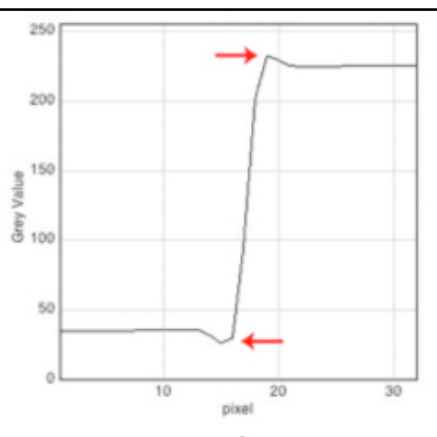

c)

Fig. 9. ESF plots obtained from images of the Slanted Edge target test: a) ESF of a raw image without edge enhancement; b) ESF of an enhanced edge avoiding the creation of artefacts; c) ESF of an over-enhanced edge with artifacts.

\subsection{Aliasing analysis}

Figure 10 corresponds to a section of a sinusoidal test target image, taken at $\mathrm{f} / 8$ with the same system and set-up as described in section 3.3. The central patch contains a sinusoidal frequency coincidental with the Nyquist camera limit. The 
left and right patches have two frequencies below and above the central one, respectively. It can be seen that, for Nyquist frequency, a colored moiré pattern is clearly visible. Besides, a slight pattern can be observed in one of the right patches, although its frequency is over the resolution capacity of the system.

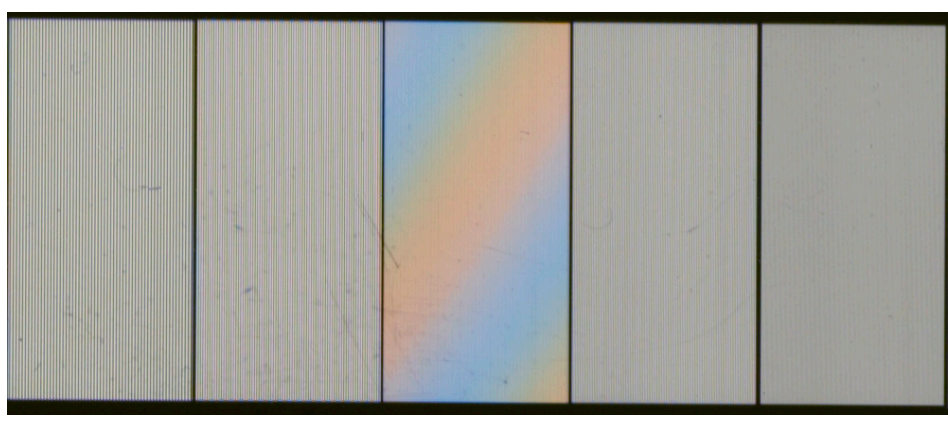

Fig. 10. Section of an image of the sinusoidal test target, corresponding to the system Nyquist frequency (central patch) and two frequencies respectively beyond (right) and below (left) it.

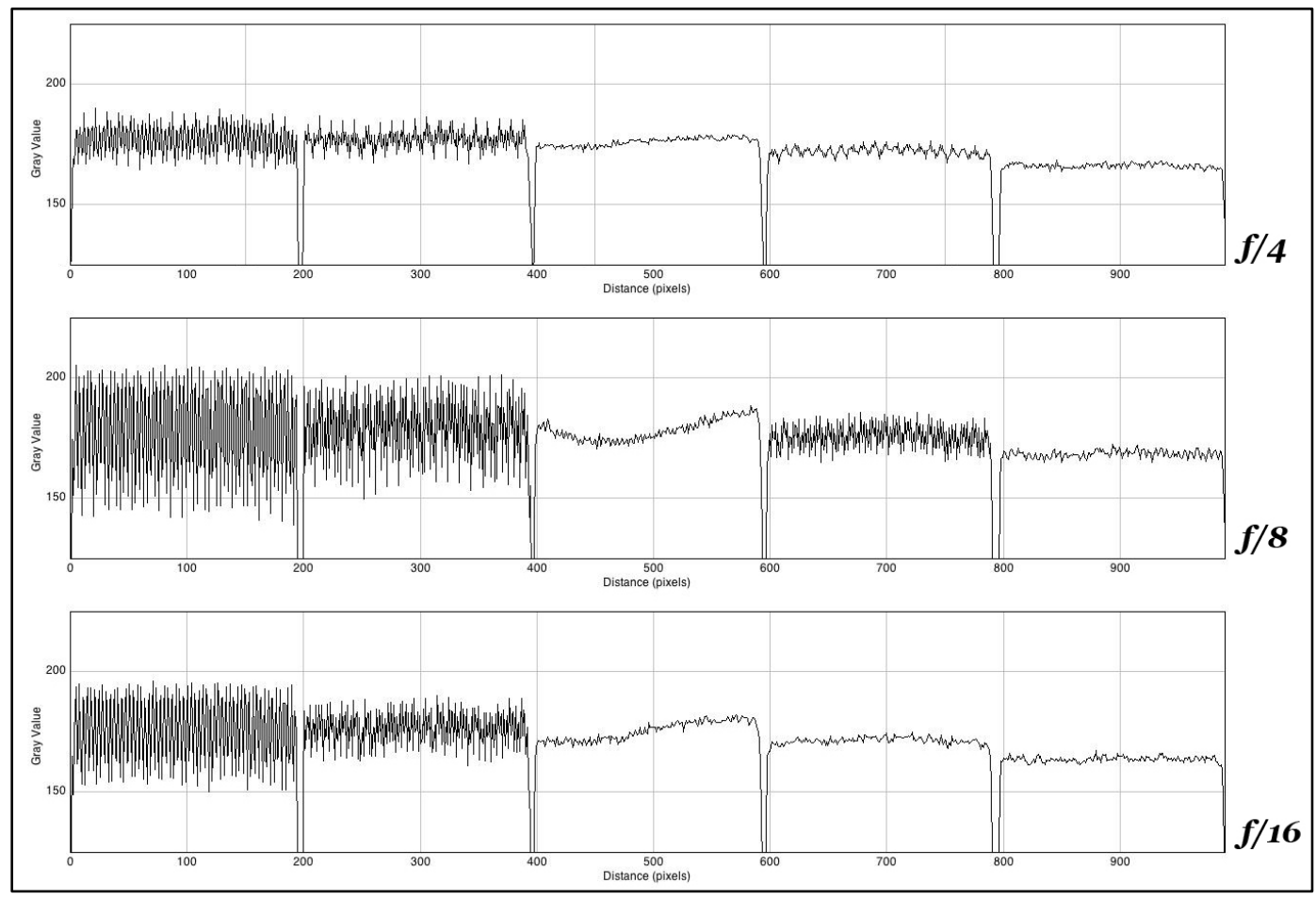

Fig. 11. Profile plots of five frequency patterns where the central one matches the camera Nyquist frequency, traced from three sinusoidal test target images. The images were taken, from above to bottom, with the diaphragm apertures of $\mathrm{f} / 4, \mathrm{f} / 8$ and $\mathrm{f} / 16$.

It is possible to confirm the presence of the aliasing effects by tracing a profile plot of this image (Figure 11), which shows the gray value for each pixel of a line selection. The central plot of the Figure 11 belongs to the image in Figure 10, while the other two have been obtained from images taken at $\mathrm{f} / 4$ and $\mathrm{f} / 16$. At $\mathrm{f} / 4$ the signal is notably reduced, probably due to the presence of residual aberrations, and at $\mathrm{f} / 16$ a similar effect is caused by diffraction. For the reproduction of original items that may be likely to cause aliasing, working with a diaphragm aperture of f/16 can significantly reduce its risk, even if the optimum apertures of the system are $\mathrm{f} / 8$ and $\mathrm{f} / 11$ (as seen in section 3.3). 


\subsection{Color management}

The color test chart has been digitized under both reflected and transmitted light conditions, using the IT8.7/2 and IT8.7/1, respectively. The two obtained images have been processed in three different ways, regarding tone curve and ICC Profile; the first one with a film curve and the generic ICC Profile (provided by the raw processing software), and the other two with a linear curve and with the generic and specific ICC profiles. In order to calculate the color accuracy achieved by each of the three options, $\Delta \mathrm{E}$ and standard deviation values, $\sigma$, have been obtained for each image, as displayed in Table 1 .

The results show that color reproduction depends on the applied ICC profile, but also on the tone curve used to process the image. In the case of applying the generic ICC profile, the use of a linear curve improves color accuracy respect to the film curve. Moreover, when a specific ICC profile is applied, the result in color reproduction is significantly better. On the other hand, this case shows that the color management applied to opaque materials achieves more accurate results than for translucent materials.

Table 1. $\Delta \mathrm{E}$ and standard deviation values obtained from two images of the IT8.7/1 and IT8.7/2 test chart, which have been captured and processed under three different conditions: film curve and generic ICC profile, linear curve and generic ICC profile, linear curve and specific ICC profile.

\begin{tabular}{|c|c|c|c|c|c|c|}
\cline { 2 - 7 } \multicolumn{1}{c|}{} & \multicolumn{2}{c|}{$\begin{array}{c}\text { Generic ICC Profile } \\
\text { Film curve }\end{array}$} & \multicolumn{2}{c|}{$\begin{array}{c}\text { Generic ICC Profile } \\
\text { Linear curve }\end{array}$} & \multicolumn{2}{c|}{$\begin{array}{c}\text { Specific ICC Profile } \\
\text { Linear curve }\end{array}$} \\
\cline { 2 - 7 } & $\Delta \mathbf{E}$ & $\sigma$ & $\Delta \mathbf{E}$ & $\sigma$ & $\Delta \mathbf{E}$ & $\sigma$ \\
\hline Reflection (IT8.7/2) & $\mathbf{1 0 , 9}$ & 5,1 & $\mathbf{7 , 8}$ & 4,7 & $\mathbf{3 , 9}$ & 1,9 \\
\hline Transmission (IT8.7/1) & $\mathbf{1 2}$ & 5,6 & $\mathbf{1 1 , 1}$ & 5,1 & $\mathbf{4 , 8}$ & 2,9 \\
\hline
\end{tabular}

\section{CONCLUSIONS}

Digitization procedures in cultural heritage digitization must take care both of lighting and capture systems. Electronic flash strobes are the most suitable illuminant. Evenness of illumination can be checked by image processing techniques. Because of its granular structure, $\mathrm{B} \& \mathrm{~W}$ negatives and color slides must be illuminated by diffuse transmitted light to avoid excessive image contrast while dyed materials as color negatives can be indifferently illuminated with specular or diffused transmitted light. Diffuse transmitted light contributes also to minimize the presence of points and scratches carried by damaged items.

Capture system characterization in terms of optical system, dynamic range, MTF measurement, aliasing analysis and color management provide valuable information relating the choice of suitable settings and operation modes.

The optical system testing, based on the identification of residual aberrations, enables to decide the suitability of a given lens and contributes to the characterization of the whole capture system.

Under the appropriate lighting conditions and with the suitable camera lens, the capturing system can be evaluated determining its OECF and the subsequent measured DR. OECF and DR calculations should be performed for different options, paying attention on the linearity of the OECF curve and on obtaining a DR higher enough to reproduce as many as possible diverse items. For photographic negatives and slides, a minimum DR covering beyond 3.0 OD units or 10EV is required.

The optimum diaphragm aperture can be established by the system MTF and, as a complementary test, by the aliasing analysis. The former provides information about the most suitable diaphragms in terms of residual lens aberrations and diffraction effects. However, for some kind of items or working magnifications that may lead to aliasing, a sinusoidal test can be useful to establish the diaphragm aperture for which diffraction contributes to minimize the moiré patterns without compromising the general sharpness of the image. In addition, the system MTF measurement provides, by means of the ESF plot, extra information that can be useful to control the application of edges enhancement at the processing or postprocessing stages 
Application of specific ICC profiles, combined with the use of a linear tone curve to process the raw file, allows achieving more accurate results in color reproduction. The evaluation of the results obtained should be done by means of measure tools that do not depend on perceptual appreciations, as the calculation of the average $\Delta \mathrm{E}$ and its standard deviation.

\section{ACKNOWLEDGEMENTS}

To Fundació Politècnica de Catalunya and to MICINN and FEDER (Project DPI2009-08879) for the financial support of this work.

\section{REFERENCES}

[1] Ray, Sidney F., “Applied Photographic Optics”, Focal Press, 2002.

[2] Allen, E., Triantaphillidou, S., "Manual of Photography", Focal Press, 2011.

[3] ISO 15739:2003. "Photography - Electronic still-picture imaging - Noise measurements"

[4] Boreman, Glenn D., "Modulation Transfer Function in Optical end Electro-Optical Systems", SPIE Press, 2001.

[5] Mitjà, C., Escofet, J., Tachó, A., and Revuelta, R., "Slanted Edge MTF measurement plug in for ImageJ", $<$ http://rsb.info.nih.gov/ij/plugins/se-mtf/index.html>

[6] Hubel, P.M., Liu, J., and Guttosch, R.J., "Spatial Frequency Response of Color Image Sensors: Bayer Color Filters and Foveon X3”, Proc. SPIE Vol. 5301, p. 402-407, 2004.

[7] Berns, Roy S., "The Science of Digitizing Paintings for Color-Accurate Image Archives: A Review", Journal of Image Science and Technology, v.45 n.4, 2001. 